\title{
La evidencia científica, la experiencia clínica y el rol de la universidad
}

\author{
Scientific evidence, clinical experience and the role of the university
}

Muchas especialidades de la odontología se han caracterizado por muchos años por estar basados en la experiencia clínica, pero eso ha ido cambiando con el tiempo. En los últimos años se le ha dado una gran importancia a la evidencia científica como la principal fuente de toma de decisiones para el tratamiento de los pacientes. No cabe duda que esto es muy importante.

En la actualidad existen innumerables revistas científicas donde podemos encontrar variada información sobre diversos temas. Si queremos saber cuál es la mejor forma de restaurar un diente después de una pulpotomía en molares primarias o después de un tratamiento de conducto en dentición permanente, tenemos que buscar artículos científicos que comparen diversas técnicas restauradoras después de estos tratamientos pulpares $(1,2)$. En estos artículos encontraremos evidencias sobre la longevidad, adaptabilidad, costo-beneficio, entre otros aspectos que podremos utilizar para la toma de decisiones. No hay duda que esta es la manera más confiable, efectiva y ética para manejar los casos de nuestros pacientes.

Sin embargo, existe aún un área gris en las profesiones médicas: aquellos conocimientos que aún no han sido estudiados a profundidad y del que tenemos tan pocas referencias por lo que es difícil aplicar los conceptos arriba mencionados. Pongamos un ejemplo. Un paciente en dentición mixta presenta mordida abierta. Hacemos una búsqueda de literatura y encontramos numerosos reportes de casos, pero escasos ensayos clínicos donde se comparen los métodos para tratar mordidas abiertas en dentición mixta $(3,4)$. La experiencia clínica y los reportes de caso nos dice que uno de los métodos para tratar la condición ha tenido éxito sistemáticamente y decidimos utilizarlo. El resultado final es un tratamiento exitoso, pero basado fundamentalmente en experiencia clínica más que en evidencia científica.

Qué importante es utilizar la evidencia científica en la toma de decisiones para el tratamiento de los pacientes y eso debe ser una obligación de los profesionales. Sin embargo, no todo ha sido estudiado a profundidad, y muchos temas solo tienen reportes de casos como nivel más alto de evidencia. En esos casos, y ante la ausencia de mejor evidencia, la experiencia clínica es muy importante. Es por ello, que es tan importante seguir buscando respuestas a aquellas preguntas que significan un beneficio para el paciente. Y esa búsqueda de respuestas debe ser a través de estudios con niveles altos de evidencia, con preguntas de investigación relevantes y directas, con diseños adecuados, y con un adecuado sistema de revisión por asesores, jurados de tesis, y editores y revisores de revistas especializadas. Las universidades tienen un rol muy importante en la búsqueda de respuestas. Una universidad no debe quedarse como una simple repetidora de conocimientos científicos que otros producen. Una 
universidad es creadora de conocimiento por excelencia. Y esos conocimientos, después de creados, deben ser necesariamente difundidos por canales que sean de alto nivel y de gran alcance.

Y aquí la importancia de tener una revista científica en nuestra universidad. La Revista Estomatológica Herediana sigue siendo una fuente importante de información científica para la comunidad odontológica del Perú y del extranjero. El objetivo de la revista debe ser recopilar las mejores investigaciones, ser muy exigentes en la revisión de los mismos, y luego hacer que estos puedan ser difundidos ampliamente. Cada uno de nosotros se convierte en un promotor para que esto sea posible, y así contribuir a la correcta difusión del conocimiento científico, brindando al clínico mayores y mejores herramientas a ser utilizadas en la toma de decisiones cada vez que tenga que tratar un paciente. Apuntemos siempre en esa dirección.

Jorge Luis Castillo-Cevallos ${ }^{1, a, b, c}$

1. Hutcheson C, Seale NS, McWhorter A, Kerins C, WrightJ.Multi-surfacecompositevsstainlesssteelcrown restorations aftermineral trioxide aggregatepulpotomy: a randomized controlled trial. Pediatr Dent. 2012; 34(7):460-7.

2. Holan G, Fuks AB, Ketlz N. Success rate of formocresol pulpotomy in primary molars restored with stainless steel crown vs amalgam. Pediatr Dent. 2002; 24(3):212-6.
3. Lentini-Oliveira DA, Carvalho FR, Rodrigues CG, Ye Q, Prado LB, Prado GF, Hu R. Orthodontic and orthopaedic treatment for anterior open bite in children. Cochrane Database Syst Rev. 2014; 9:CD005515.

4. Lentini-Oliveira DA, Carvalho FR, Qingsong Y, Junjie L, Saconato H, Machado MA, Prado LB, Prado GF. Orthodontic and orthopaedic treatment for anterior open bite in children. Cochrane Database Syst Rev. 2007 Apr 18; (2):CD005515. 